\title{
Deep Brain Stimulation in Huntington's Disease: The Current Status
}

\author{
Dagmar Zeef*, Frédéric Schaper, Rinske Vlamings, Veerle Visser-Vandewalle and Yasin Temel
}

\begin{abstract}
Departments of Neuroscience and Neurosurgery, School for Mental Health and Neuroscience (MHeNS), Maastricht University and Maastricht University Medical Center, Maastricht, The Netherlands
\end{abstract}

\begin{abstract}
Background: Huntington's disease (HD), a progressive neurodegenerative disorder, is characterized by choreic movements and psychiatric changes. Within 10 to 20 years after the onset of the disease most patients die of disease related complications. Until now, no effective therapies have been found for this devastating disease. Deep brain stimulation (DBS) is a promising new symptomatic treatment for HD. In the last decade, theories on the potential benefit of DBS in HD have been made and different groups have explored this in case studies.
\end{abstract}

Objectives: In this paper we provide an overview of the result obtained with DBS in patients with HD.

Results: The studies performed thus far are case studies. Their results suggest that DBS of the globus pallidus internus is safe and well-tolerated, and reduces choreic movements, without substantial effects on psychiatric functions.

Conclusions: DBS in HD should still be seen as experimental. The data published until now, demonstrate a therapeutic effect on refractory motor symptoms and justify further investigation.

Keywords: Huntington's disease, deep brain stimulation.

\section{INTRODUCTION}

Huntington's disease (HD) is first described by George Huntington in 1872 [1]. The disease is characterized by adult-onset of symptoms, which comprise cognitive decline and personality changes. These are followed by generalized motor symptoms with chorea being the most well known feature of the disease. Within 20 years after the onset of the disease most patients die of complications, such as dysphagia, aspiration, and overall exhaustion [2]. Until now, no effective therapies have been found [3, 4].

$\mathrm{HD}$ is a neurodegenerative disorder caused by a CAG repeat expansion in the gene encoding for the Huntingtin protein (IT-15). CAG is a trinucleotide coding for glutamine, repeats result in a polyglutamine tract. Huntingtin is expressed throughout the body, with high levels in the brain. Here, it is mainly expressed within the neocortex, cerebellum, striatum and hippocampus. In the non-effected population the amount of CAG repeats of the gene varies amongst 6-35. When this number exceeds 36 , the disease inducing Huntingtin protein is being formed. The length of CAG repeats seams to correlate with the age of onset: CAG repeats over 60 are associated with childhood onset; Juvenile HD [5].

The CAG repeat expansion leads to atrophy and cell death within the striatum and, to a lesser extent, in the cerebral cortex. The striatum is the most important motor input structure of the basal ganglia. Besides the basal ganglia comprise the pallidum (external and internal parts), substantia nigra and the subthalamic nucleus (STN). The striatum

*Address correspondence to these authors at the UNS 50, Box 38, 6229 ER Maastricht, The Netherlands; Tel: +31 - 43 - 38811 74; Fax: +31 - 43 - 367 10 96; E-mail: dh.zeef@ maastrichtuniversity.nl receives 'motor' input from the cerebral cortex and 'limbic' input from the hippocampus and the amygdala. These input structures form together with the basal ganglia and the thalamus, the cortico-basal ganglia-thalamo cortcial circuits: motor, oculomotor, two prefrontal (dorsolateral and lateral orbitofrontal) and the limbic circuits [6]. Cell damage in the cortex and striatum in HD, results in dysfunction of the cortico-basal ganglia-thalamo cortical circuits.

Within the striatum the most affected cells are the medium spiny neurons, which can be subdivided in "early-" and 'late-affected' cells. Among the 'early-affected' cells are the 'indirect' pathway neurons and the 'late-affected' cells contain the 'direct' pathway neurons [7]. Eventually the specific cell loss spreads to several brain regions; the pallidum, STN, substantia nigra, cerebellum and thalamus.

The above-described loss of neurones in the striatum within the 'indirect' pathway, is thought to lead to an impaired neuronal activity of the external segment of the globus pallidus (GPe) and related changes in other basal ganglia structures [8]. Progression of disease leads to loss of the 'direct' pathway neurons responsible for neuronal activity changes in the globus pallidus internus (GPi).

Deep brain stimulation (DBS), nowadays a widely applied neurosurgical therapy, is one of the methods used to correct impaired neuronal firing patterns [9]. In Parkinson's disease, this therapy has proven to be effective in the advanced stage of the disease [10]. DBS is especially effective in disorders with a known dysfunctional brain region. In HD, the pallidal complex has been proposed as a target for DBS and consequently its effects are explored.

Thus far, several groups have performed DBS in HD patients. All studies are case-reports. Here, we review these 
studies structurally and discuss the current status of DBS in the treatment armamentarium for HD.

\section{LITERATURE REVIEW}

To our knowledge, the first publication of DBS in HD is by Moro et al. in 2004 [11]. They described a 43-year-old man with genetically confirmed HD. His symptoms included severe and disabling chorea, dysarthria, dysphasia, balance impairments with falls, and weight loss. Cognitive alterations were not described. The patient was selected for DBS, as his motor symptoms became refractory to medication. The GPi was chosen as DBS target based on previous results obtained in Parkinson's disease. In Parkinson's disease treatment with 1-dopa causes dyskinesia, a hyperkinesia like chorea, which can be treated with pallidal surgery [12]. Moro and associates hypothesized that stimulation of the GPi would reduce choreic movements in HD patients.

Pre- and postoperative the patient was scored with use of the Unified Huntington's Disease Rating Scale (UHDRS) (Table 1). The scoring was performed in a double-blind manner for stimulation frequency. Bilateral stimulation of the GPi with a stimulation frequency of $40 \mathrm{~Hz}$ induced a significant decline of choreic movements and dystonia along with a slight reduction of bradykinesia. When stimulation frequency was raised to $130 \mathrm{~Hz}$ the patient experienced further improvement in chorea and dystonia, but bradykinesia and other hypokinetic symptoms worsened. Moro et al., compare this finding to unpublished data of J. L. Vitek and M.R. DeLong (personal communication to Dr. Moro) who observed worsening of bradykinesia in HD patients after GPi pallidotomy. Finally, high doses of tetrabenzine used by the patient preoperatively could be reduced with $50 \%$ and haloperidol and amantadine could be stopped. Neuropsychological outcome parameters are not mentioned. In the end the authors describe an overall improvement of daily life.

Two years later Hebb et al. [13], described a 41-year-old man with HD with a disease duration of 13 years. His motor symptoms had progressed to severe choreathetosis and disabling dystonia of all extremities, head and trunk. Furthermore, neuropsychological evaluation revealed memory deficits, but no severe psychiatric disorders. As his symptoms became refractory to drug treatment, DBS was performed as a last resort therapy. The GPi was chosen as stimulation target based on results reported by Moro et al. [11]. Pre- and post operatively the UHDRS was used to determine stimulation frequencies. After a recovery period of one-month high frequency stimulation, $180 \mathrm{~Hz}$, was initiated. The authors do not give a rationale for choosing this frequency and refer to Moro et al. [11], who stimulated with $130 \mathrm{~Hz}$. Nonetheless, a substantial reduction of chorea was established with DBS. Furthermore a general improvement of motor symptoms (Table 1) occurred without reporting an increase in hypokinesia, which was seen in the previous study [11]. A trial with low frequency, $40 \mathrm{~Hz}$, showed poor control of chorea and high frequency stimulation was restarted. After 10 months of stimulation with $180 \mathrm{~Hz}$, the patient experienced impairment of his glottic function, which did not improve by changing the stimulation settings, and led to institutionalization. At the last follow-up time-point, 12 months postoperatively, GPi DBS still adequately treated the patient's chorea, but rigidity and dysphagia progressed. Even so, both the patient as his family reported evident improvement of the quality of life since GPi DBS. According to the authors, neuropsychologial parameters were not changed postoperatively.

In 2008, two case-reports of DBS in HD were reported. Fasano et al. [14], reported results of GPi DBS in a 72-yearold man with HD. The patient was suffering for 17 years of severe progressive generalized choreathetosis and cognitive decline. As medication could only give modest improvement of symptoms, GPi DBS was performed. For evaluation, the UHDRS was obtained pre- and postoperatively in a doubleblind manner to determine the stimulation frequency. Stimulating with a frequency of $40 \mathrm{~Hz}$ gave a significant reduction of choreathetosis, which was further improved by increasing the frequency to $130 \mathrm{~Hz}$. Unfortunately, stimulation with 130 $\mathrm{Hz}$ worsened hypokinetic symptoms. After 11 months, stimulation was stopped due to progressive gait impairments and apathy. Nevertheless, chorea remained suppressed and with the introduction of 1-dopa gait impairment was improved. During the follow-up period, general cognitive decline was noticed. In the same year, Biolsi and co-workers [15] described their 4-year follow-up findings in a HD patient treated with GPi DBS. The patient, a 60-year-old man, had been suffering from HD symptoms for over 10 years. Preoperative symptoms included severe choreic movements in the face, neck, trunk, upper and lower extremities and oromandibular hyperkinesias. Furthermore, examination showed hypotonia of the extremities, but no general bradykinesia or dystonia. Neuropsychiologic testing revealed moderate cognitive dysfunction, without influencing the ability to perform daily activities. DBS was started with a frequency of $130 \mathrm{~Hz}$ and led within 10 days postoperatively to a significant reduction of his chorea but worsened bradykinesia. Three weeks after stimulation, 1-dopa was introduced to treat bradykinesia. After a follow-up period of 4 years there was a stable reduction of chorea under influence of DBS. When the stimulation was switched off, there was an immediate and severe worsening of chorea in all extremities, trunk and face. In addition autonomous gait and standing became impossible; the patient had a limited function of his hands and failed to articulate correctly. These symptoms all improved when the stimulator was turned on again. Postoperative neuropsychological testing showed no cognitive changes when compared to the preoperative status.

Recently (2010) Kang et al. [16], published their findings of GPi DBS in two patients with HD. Both patients suffered from severe chorea and balance impairments, which could not be controlled with drug therapy. In addition, both patients suffered from depression and neuropsychological evaluation showed mild cognitive impairment. Pre- and postoperative UHDRS (Table 1) showed a decline in chorea for both patients. The first patient experienced a reduction in chorea already before stimulation was initiated. However this reduction has not been formally rated with the UHDRS. Stimulation was initiated 6 months post-operatively and the UHDRS was assessed with high and low frequency stimulation $(130 \mathrm{~Hz}$ and $40 \mathrm{~Hz}$, respectively). Remarkably, the UHDRS scores in off- and on-conditions showed no noticeable difference. The authors mention the appearance of chorea when the stimulator was turned off without the awareness of the patients, but exact data from this observation is lacking. 


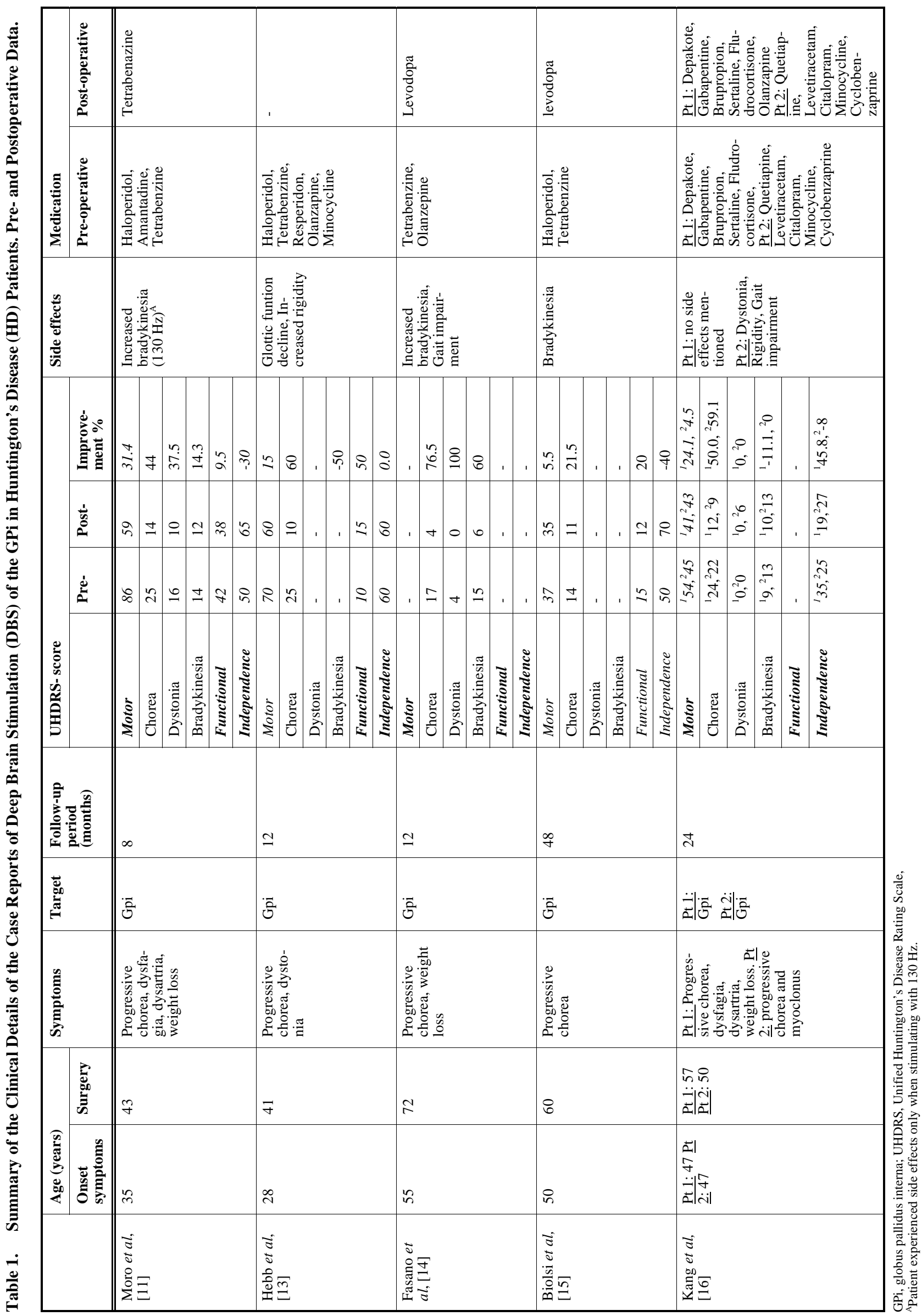


The second patient had a mild reduction of his chorea postoperatively, which further improved when stimulation was initiated. When stimulation was turned off the patient experienced severe chorea within minutes. Unfortunately, the patient's dystonia, rigidity and gait impairment increased in the two-year follow-up period. Of note, Kang et al. [16], performed a staged bilateral GPi electrode implantation (3 to 6 months after the first implantation). This was done to observe the effect of unilateral electrode implantation on their motor symptoms and whether serious adverse side effects occurred. As both patients experienced slight decline of unilateral chorea and experienced no (mentioned) complications, the contralateral operation was carried out. Neuropsychological evaluations of both patients showed a slight decline in cognitive functions during the follow-up period, which was attributed to disease progression.

\section{DISCUSSION}

To evaluate whether DBS has a place in the treatment of $\mathrm{HD}$, we systematically looked at the results of the case reports published until now. The overall outcome of the patients reported shows that DBS might improve motor symptoms. The long-term follow-up study by Biolsi et al. [15], shows, that 4 years after the initiation of GPi-DBS, there is still a significant improvement of the patient's chorea and no worsening of other symptoms during stimulation. However, these results contrast the short-term follow-up studies $(<12$ months), which show, in addition to a reduction of the chorea due to DBS, worsening of gait, balance and cognitive functions $[13,14]$. Low frequency stimulation leads to a reduction of chorea. Initiation of high frequency stimulation aggravated this effect. The overall side effect of high frequency stimulation was worsening of hypokinesia, which can also be a feature of disease progression. An interesting but contrasting finding is that despite the cessation of the stimulation, the chorea did not return in one study [14] and did return in another study [15].

In addition to the motor symptoms, some brief neuropsychological information of the patients was given. Evaluation of these results is difficult because of lack of details on methods and outcomes.

The DBS target used in the case reports is the GPi. Other targets within the basal ganglia such as the GPe and STN $[17,18]$ have been suggested as potential stimulation targets. These hypotheses are based on previous research, mainly Parkinson's disease related research. Another interesting theory proposes the substantia nigra parc compacta (SNc) as a potential target. Bonelli et al. [17], report spontaneous decline of choreic movements in a patient with HD. In a later publication they state that the disappearance of chorea could be linked to the atrophy of the SNc and suggest DBS of this area [17]. Interestingly, in a recent study with transgenic HD rats, authors found increased number of dopaminergic cells in the SNc and linked this to chorea [19]. Altogether, the last word about the most optimal target for DBS has not been spoken.

In conclusion, DBS in HD should still be seen as experimental. The data published until now, however, demonstrate a therapeutic effect on refractory motor symptoms and justify further investigation.

\section{REFERENCES}

[1] Huntington G. On Chorea. The Medical and Surgical Reporter: A Weekly Journal (Philadelphia: SW Butler) 1872; 26(15): 317-21.

[2] Walker FO. Huntington's disease. Lancet 2007; 369(9557): 218-28.

[3] Bonelli RM, Wenning GK, Kapfhammer HP. Huntington's disease: present treatments and future therapeutic modalities. Int Clin Psychopharmacol 2004; 19(2): 51-62.

[4] Adam OR, Jankovic J. Symptomatic treatment of Huntington disease. Neurotherapeutics 2008; 5(2): 181-97.

[5] MacDonald ME, Ambrose CM, Duyao MP, et al. A novel gene containing a trinucleotide repeat that is expanded and unstable on Huntington's disease chromosomes. Cell 1993; 72(6): 971-83.

[6] Temel Y, Blokland A, Steinbusch HW, Visser-Vandewalle V. The functional role of the subthalamic nucleus in cognitive and limbic circuits. Prog Neurobiol 2005; 76(6): 393-413.

[7] Sieradzan KA, Mann DM. The selective vulnerability of nerve cells in Huntington's disease. Neuropathol Appl Neurobiol 2001; 27(1): $1-21$

[8] Joel D, Ayalon L, Tarrasch R, Veenman L, Feldon J, Weiner I. Electrolytic lesion of globus pallidus ameliorates the behavioral and neurodegenerative effects of quinolinic acid lesion of the striatum: a potential novel treatment in a rat model of Huntington's disease. Brain Res 1998; 787(1): 143-8.

[9] Benazzouz A, Hallett M. Mechanism of action of deep brain stimulation. Neurology 2000; 55 (12 Suppl 6): S13-6.

[10] Wichmann T, Delong MR. Deep brain stimulation for neurologic and neuropsychiatric disorders. Neuron 2006; 52(1): 197-204.

[11] Moro E, Lang AE, Strafella AP, et al. Bilateral globus pallidus stimulation for Huntington's disease. Ann Neurol 2004; 56(2): 2904.

[12] Lang AE, Lozano AM, Montgomery E, Duff J, Tasker R, Hutchinson W. Posteroventral medial pallidotomy in advanced Parkinson's disease. N Engl J Med 1997; 337(15): 1036-42.

[13] Hebb MO, Garcia R, Gaudet P, Mendez IM. Bilateral stimulation of the globus pallidus internus to treat choreathetosis in Huntington's disease: technical case report. Neurosurgery 2006; 58(2): E383; discussion E.

[14] Fasano A, Mazzone P, Piano C, Quaranta D, Soleti F, Bentivoglio AR. GPi- DBS in Huntington's disease: results on motor function and cognition in a 72- year-old case. Mov Disord 2008; 23(9): 1289-92.

[15] Biolsi B, Cif L, Fertit HE, Robles SG, Coubes P. Long-term follow-up of Huntington disease treated by bilateral deep brain stimulation of the internal globus pallidus. J Neurosurg 2008; 109(1): 130-2.

[16] Kang GA, Heath S, Rothlind J, Starr PA. Long-term follow-up of pallidal deep brain stimulation in two cases of Huntington's disease. J Neurol Neurosurg Psychiatry 2011; 82: 272-77.

[17] Bonelli RM, Koltringer P, Kenner L, Reisecker F. Reversible Huntington's disease? Lancet 1998; 352(9139): 1520-1.

[18] Bonelli RM, Gruber A. Deep brain stimulation in Huntington's disease. Mov Disord 2002; 17(2): 429-30.

[19] Jahanshahi A, Vlamings R, Kaya AH, et al. Hyperdopaminergic status in experimental Huntington disease. J Neuropathol Exp Neurol 2010; 69(9): 910-7. 